\title{
On the Impact of Traffic Characteristics on Radio Resource Fluctuation in Multi-Service Cellular CDMA Networks
}

\author{
Keivan Navaie \\ Sys. and Comp. Department \\ Carleton University, Ottawa, Canada \\ keivan@sce.carleton.ca
}

\author{
Ahmad R. Sharafat \\ Electrical Eng. Department \\ TMU, Tehran, Iran \\ sharafat@isc.iranet.net
}

\author{
Yiqiang Q. Zhao \\ School of Math. and Stats. \\ Carleton University, Ottawa, Canada \\ zhao@math.carleton.ca
}

\begin{abstract}
In this paper, we study the effect of users' traffic characteristics on the temporal behavior of total downlink interference in multi-service wireless CDMA networks. We show that under certain conditions on traffic characteristics that cover a range of practical situations, downlink interference follows an asymptotically self-similar process and thus is long-range dependent. We then obtain the asymptotic outage probability and show that in a network that is designed based on the Poisson assumptions the outage probability is degraded by the interference long-range dependent. Simulation results for actual cases confirm analytical results.
\end{abstract}

\section{INTRODUCTION}

Future personal communication systems support multiservices including multimedia, voice, and data. A promising air interface technology for future wireless cellular communication networks is Code Division Multiple Access (CDMA). Since CDMA systems have been shown to be interferencelimited [1], multiple-access interference plays an important role in the performance analysis of such systems and is usually considered as radio resource indicator. Therefore, proper utilization of more accurate information on the temporal behavior of the interference can lead to the development of effective resource control mechanisms. However, the conventional approaches for modeling interference in wireless CDMA only use the marginal distribution of the total interference [1], [2], [3], therefore do not consider its temporal behavior.

In this paper, we consider such temporal behavior for heterogeneous services in wireless CDMA networks, which can be utilized effectively in designing radio resource control mechanisms such as outer-loop power control, call admission control, and load balancing. These control mechanisms operate in substantially longer time periods (i.e., from a few milliseconds to seconds) compared to other mechanisms in the lower layers including modulation, detection and fast power control. In the considered time scales, several system parameters in different network layers cause temporal fluctuation of interferences. These include, for instance, the total number of active users (calls), their call/session durations, the allocated power to each call/session in the corresponding base-station (BS), channel variations and user mobility. We develop a model that frames the effect of traffic characteristics on the downlink interference over long time scale. In our approach the traffic characteristics at the application layer and the information on the wireless channels are combined in a cross-layer model to characterize the downlink interference.

Traffic modeling for voice-only CDMA cellular networks is based on a Poisson process (see e.g. [1], [4]) that has its root in telephony networks (for a comprehensive review see [5]). They assume a Poisson process: calls arrive independently over time at a constant rate while their corresponding call durations have exponential distributions. This model is still almost invariably used to model the arrivals and departures of calls in the network, even though it may not be an adequate model for call/packet durations of multi-media and data traffics with heavy tail distributions [6]. Therefore, the impact of traffic characteristics on the downlink interference in a heterogeneous cellular network is different from that of voice-only networks. The effect of non-Poisson traffic on the performance of wireless network was first addressed in [7], where the requirements for supporting such traffic were examined. It was shown in [8] that the capacity of a CDMA cellular network, designed for Poisson traffic, is substantially reduced when the input traffic has long-range dependence.

The effect of packet data traffic on the interference in a datacentric packet-based CDMA network has been studied in [9], [10]. They ignore the effects of call admission procedure and use a method in [11] together with basic notions of a heavy-tail process to explain the self-similarity in total interference that is a direct consequence of data users' traffic characteristics. Obviously their method is not applicable to multi-service CDMA cellular networks that support both packet-based and connection-oriented services. They also assume a very specific wireless channel that may not be applicable to actual cases.

We use the cross-layer model in [12] to characterize the downlink interference that is a function of channel autocovariance, call/packet-duration distribution, call/packet arrivals, and the auto-correlation of the allocated power to each call/packets. In [12] we show that in a multi-cell network, for heavy-tail distribution of call durations, the corresponding interference follows an asymptotic self-similar (as-s) process. The respective heavy-tail distribution depends on call durations, bit-rate variations, and channel characteristics. Selfsimilar processes are used to characterize long-range dependence in the network traffic (see e.g. [6]).

We then study the effect of self-similarity in the downlink interference on the outage probability as an indicator of 
the cellular network performance. We consider the outage probability as the tail probability of an $M / M / \infty$ buffer-less multiplexer [1], [4], [13]. We obtain the asymptotic outage probability as a function of self-similarity index using the results in [14].

We simulate a heterogeneous wireless network and observe self-similarity in the downlink interference when the call duration-of at least one service has a heavy-tail distribution. Simulation results also confirm that the outage probability increases as a consequence of interference long-range dependence.

Organization of this paper is as follows. The interference model is presented in Section II. In Section III, we present the analytical results. Simulation results are presented in Section IV. The concluding remarks are presented in Section V.

\section{INTERFERENCE MODEL}

Here we restate the model used in [12] for total downlink interference. Total downlink interference, $I(n)$, for a considered user, is a weighted sum of the transmitted power of BSs, $P^{c}(n)$, for $n \in \mathbb{Z}, \mathbb{Z}=\{\ldots,-1,0,1, \ldots\}$ and $c=1, \ldots, N_{C}$, where $N_{C}$ is the number of cells in the network

$$
I(n)=\sum_{c=1}^{N_{C}} \xi^{c}(n) P^{c}(n) g^{c}(n) .
$$

The weighted coefficients are the corresponding channel gains, $g^{c}(n)$, and average normalized cross-correlation, $\xi^{c}(n)$, between the user's signal and the received signals of other users are stationary processes independent of $P^{c}(n)$. Each sample of $I(n)$ is valid over a window of length $T_{w}$ seconds that is the time scale of modeling. We assume that $T_{w} \gg T_{c}$, where $1 / T_{c}$ is the spreading bandwidth of the cellular CDMA network. In (1) we consider $I(n)$ as the interference received in a user in cell 1 , therefore we assume that the power allocated to the considered user is not included in $P^{1}(n)$.

To study $I(n)$, we assume that a regular power transmission regime is applied network-wide, in which the transmitted power by any BS is not substantially higher than the transmitted power by other BSs. This assumption is valid if a load balancing mechanism is applied.

In the time slot $n$, each BS serves a set of active users (calls) in its coverage area, therefore the transmitted power by the $\mathrm{BS} c, P^{c}(n)$, is the sum of allocated powers to all calls in the corresponding coverage area

$$
P^{c}(n)=\sum_{j=1}^{J} \sum_{i \in \mathbb{N}} p_{j i}^{c}\left(n-v_{j i}^{c}+1\right),
$$

where $J$ is the number of services provided by the network, $\mathbb{N}=\{1,2, \ldots\}, p_{j i}^{c}($.$) is the allocated power of call i$ of service $j$ in cell $c$, and $v_{j i}^{c} \in \mathbb{Z}$ is the start time of the $i^{\text {th }}$ call in cell $c$ that receives service $j$. Calls are enumerated by $i$ in the order of their arrival, such that in each cell $c, v_{j i}^{c} \leq v_{j i+1}^{c}$. For the $i^{\text {th }}$ call of service $j$ in cell $c$ with a call duration of $\tau_{j i}^{c} \in \mathbb{N}$ seconds, $p_{j i}^{c}($.$) is the allocated power in its call duration, and$ is equal to zero otherwise.
To characterize $I(n)$, we first need to obtain the characteristics of $P^{c}(n)$ and $g^{c}(n)$. We assume that for each given cell $c$ and service $j$, the call duration sequence process $\left\{\tau_{j i}^{c}, i \in \mathbb{N}\right\}$, the new call arrival rates sequence process $\left\{\mu_{j i}^{c}(),. i \in \mathbb{N}\right\}$, and the allocated power sequence process $\left\{p_{j i}^{c}(),. i \in \mathbb{N}\right\}$ are independent and identically distributed (i.i.d.) random processes for all $i \in \mathbb{N}$. We denote $\tau_{j i}^{c}, \mu_{j i}^{c}(n)$ and $p_{j i}^{c}(n)$ by the generic random variables $\tau_{j}^{c}, \mu_{j}^{c}(n)$ and $p_{j}^{c}(n)$, respectively.

In this model, the traffic characteristics of a user of service $j$ is specified by three processes, $\mu_{j}^{c}(n), \tau_{j}^{c}$ and $p_{j}^{c}(n)$, where $p_{j}^{c}(n)$ is a function of the service type $j$, the bit-rate, and the power allocation strategy in the network. In [12] we show that the downlink interference can be completely specified by characterization of the traffic characteristics corresponding to different services provided by the network and channel processes, $g^{c}(n)$, for all $c$. In the followings we briefly review the models we use in this paper for new call arrival process, call duration process, allocated power process and wireless channel process.

1) New Call Arrivals: Assuming the arrival rates of new calls for each service type are less than the values for which the network was designed, in [12] we show that using a regular interface-based call admission control, a Poisson distribution is an appropriate model for call arrivals $\mu_{j}^{c}(n)$,

$$
\operatorname{Pr}\left\{\mu_{j}^{c}(n)=\nu\right\}=\frac{\left(\lambda_{j}^{c}\right)^{\nu} e^{-\lambda_{j}^{c}}}{\nu !},
$$

where $\lambda_{j}^{c}$ is the arrival rate.

2) Call Durations: We denote both packet duration and call duration as "call duration" and assume an arbitrary distribution for the call duration. For voice service an exponentially distributed call duration is assumed as in [1]. For non-voice traffics a general heavy-tail distribution is considered (see e.g. [6]). A random variable $X$ is called heavy-tailed with infinite variance, if there exist a slowly varying function $L(x)$ such that

$$
P(|X| \geq x) \sim L(x) x^{-\kappa} \quad x \rightarrow \infty
$$

where $0<\kappa<2$ and the symbol ' $\sim$ ' means behaves asymptotically as (i.e. $\phi(k) \sim \varphi(k)$ means: $\lim _{k \rightarrow \infty} \frac{\phi(k)}{\varphi(k)}=$ 1). A function $f(x)>0, x \in \mathbb{R}$ is called a slowly varying function if for all $u \in \mathbb{R}_{+}, \frac{f(u x)}{f(x)} \rightarrow 1$, as $x \rightarrow \infty$.

An example of a heavy tail distribution is Pareto distribution

$$
\operatorname{Pr}\{\tau=l\}=\eta_{0} l^{-\alpha-1},
$$

where $1<\alpha<2, l \in \mathbb{N}$ and

$$
\eta_{0} \triangleq \frac{1}{\sum_{l=1}^{\infty} l^{-\alpha-1}} .
$$

Pareto distribution has been used to model call durations (see e.g. [15]).

3) Allocated Power to Each Call: For $p_{j}^{c}(n)$, we note that for a given channel, the allocated power to a given user at time slot $n$ is generally a concave function of its bit-rate [1]. 
4) Wireless Channel: We assume that $g^{c}(n)$ is a secondorder stationary process. The auto-covariance function of $g^{c}(n)$ is denoted by $C_{g}^{c}(k)$. We also assume that

$$
C_{g}^{c}(k) \sim L_{g}^{c}(k) k^{-\beta_{g}^{c},} \quad k \rightarrow \infty
$$

where $k$ denotes time with a temporal resolution $T_{w}, L_{g}^{c}(k)$ is a slow varying function and $\beta_{g}^{c}>0$ is the channel autocovariance decay exponent. For the corresponding time scales, in [12] we show that this model is consistent with slow fading processes.

\section{AnALyticAl Results}

We show that the total downlink interference in multiservice wireless CDMA networks follows an asymptotically self-similar process an thus is long range dependent. The selfsimilarity emanates from users traffic characteristics. First we define an asymptotically self-similar and long range dependent processes.

Definition 1: Asymptotically self-similar process [15] A real-valued second-order stationary random process $I=$ $(\ldots, I(-1), I(0), I(1), \ldots)$ is called asymptotically selfsimilar process (as-s), with self-similarity index $H=1-\beta / 2$, $0<\beta<1$, if

$\lim _{m \rightarrow \infty} C_{(m)}(k)=\frac{C_{(m)}(0)}{2}\left((k+1)^{2-\beta}-2 k^{2-\beta}+(k-1)^{2-\beta}\right)$,

where $k \in \mathbb{Z}_{+}, C_{m}(k)$ is the auto-covariance function of $I^{m}$ that is the average process of $I$ over blocks of length $m$.

A process $I$ is as-s if the correlation coefficients of the average process of block length $m$ as $m \rightarrow \infty$ are identical to those of a self-similar process. A sufficient condition for a second-order stationary process $I$ to be asymptotically selfsimilar is that for $k \in \mathbb{Z}_{+}, k \rightarrow \infty$, the auto-covariance function of $I, C(k)$, behaves asymptotically as $L(k) k^{-\beta}$, (i.e. $\left.C(k) \sim L(k) k^{-\beta}\right)$, in which $0<\beta<1$, and $L(k)$ is a slowly varying function [15].

Definition 2: Long Range Dependent Process [16] A stationary process $I$ is long range dependent if there exists a real number $0<H<1$ such that for $k \rightarrow \infty$ the correlation of $I, \rho_{I}(k)$ satisfies:

$$
\rho_{I}(k) \sim k^{2 H-2} .
$$

An asymptotically self-similar process is long-range dependent.

\section{A. Interference Self-similarity}

Suppose that the downlink interference process, $I=$ $(\ldots, I(-1), I(0), I(1), \ldots)$, is a finite-mean, finite-variance second-order stationary process. In the following proposition, we derive the necessary conditions on traffic and channel characteristics under which the downlink interference is an as-s process.
Proposition 1 [12]: Consider the downlink interference process, $I$, and let $\beta_{P}^{c}, c=1, \ldots, C$, satisfy

$$
\sum_{j=1}^{J} \lambda_{j}^{c} \operatorname{Pr}\left\{\tau_{j}^{c}=k\right\} r_{j(k)}^{c}(k) \sim L_{P}^{c}(k) k^{-\beta_{P}^{c}-2}, k \rightarrow \infty,
$$

where $L_{P}^{c}(k)$ is a slowly varying function. Now, $I$ is an as-s process with self-similarity index $H=1-\beta^{*} / 2$ if there exists at least one $c$ such that $0<\beta_{P}^{c}<1$ or $0<\beta_{g}^{c}<1$, and

$$
\beta^{*}=\min _{c} \min \left\{\beta_{P}^{c}, \beta_{g}^{c}\right\}
$$

Self-similarity is a phenomenological model for interference that can be specified by a small number of parameters. Selfsimilar behavior of the downlink interference is in sharp contrast with the conventional Poisson-based traffic models used to model the downlink interference. Here we show the outage probability in a network that is designed based on the Poisson based model would be degraded in the presence of self-similarity of downlink interference.

\section{B. Effect of interference self-similarity on the outage proba-} bility

CDMA systems have been shown to be interference-limited. It is simple to show that outage can be modelled as the tail probability of a $M / M / \infty$ queue when the stochastic model of the interference can be evaluated [1], [4], [13]

$$
P_{\text {outage }}=\operatorname{Pr}\left\{I>I_{t h}\right\} \text {, }
$$

where $I$ is the interference level and $I_{t h}$ is the interference threshold. For the interference process which is stationary and short-range dependent, the outage probability which is modelled as tails of the distribution of the queue length should satisfy

$$
P_{\text {outage }} \approx e^{-\delta I_{t h}}
$$

for some positive $\delta$ that is a function of cellular network parameters [14]. The asymptote in (9) holds in great generality. For self-similar interference we obtain the asymptotic outage probability in the following Proposition.

Proposition 2: The outage probability for self-similar interference with self-similarity index $H$ and maximum threshold level $I_{t h}$ is

$$
P_{\text {outage }} \approx e^{-\delta I_{\text {th }}^{2(1-H)}} .
$$

Proof outline: Proposition 2 is proved using Corollary 2.3 in [14] that gives the distribution of the interference with constant $I_{s}>E I$ where $I_{s}$ is the interference level corresponding to the service rate provided by the base-station to active calls

$$
\lim _{I_{t h} \rightarrow \infty} b^{-2(1-H)} \log \operatorname{Pr}\left\{I>I_{t h}\right\}=-\delta,
$$

where

$$
\delta=\inf _{c>0} c^{-2(1-H)} \lambda^{*}\left(c+I_{s}\right)
$$


TABLE I

Simulation PARAMETERS

\begin{tabular}{|l|l|}
\hline Parameter & Interpretation \\
\hline Number of BSs & 19 \\
\hline Cell Radius & $100 \mathrm{~m}$ \\
\hline BSs Transmit Power & $10 \mathrm{~W}$ \\
\hline Physical Layer & Based on UMTS \\
\hline Power Control & Fast Power Control 1500/s \\
\hline$T_{w}$ & $10 \mathrm{~ms}$ \\
\hline Standard Deviation of Fading & $8 \mathrm{~dB}$ \\
\hline Loss Exponent & -4 \\
\hline$T_{f}$ & $100 \mathrm{msec}$ \\
\hline$E \xi^{1}$ & 0.5 \\
\hline Services & $12.2 \mathrm{kbps}$ voice, 32 and $64 \mathrm{kbps}$ data \\
\hline 12.2 kbps voice & $E_{b} / I_{0}=5 \mathrm{~dB}, 5$ Erlangs \\
\hline 32 kbps data & $E_{b} / I_{0}=3 \mathrm{~dB}$ \\
& Pareto Dist., $\alpha_{1}=1.5, E \tau_{1}=2 \mathrm{~s}$ \\
\hline 64 kbps data & $E_{b} / I_{0}=2 \mathrm{~dB}$ \\
& Pareto Dist., $\alpha_{2}=1.8, E \tau_{2}=1.5 \mathrm{~s}$ \\
\hline
\end{tabular}

and $\lambda^{*}(x)$ is the Legendre transform of $\lambda(x)$,

$$
\lambda^{*}(x)=\sup _{\theta}\{\theta x-\lambda(\theta)\}
$$

and $\lambda(\theta)=\lim _{n \rightarrow \infty} \lambda_{n}(\theta)$ where

$$
\lambda_{n}(\theta) \triangleq n^{-2(1-H)} \log E e^{\theta n^{1-2 H}} \sum_{k=1}^{n} I(k) . \square
$$

Note that for $H=1 / 2,(9)$ and (10) are the same. It is simple to show that for a given set of network parameters $P_{\text {outage }}$ is increased as a consequence of self-similarity in total interference.

\section{Simulation Results}

We consider a two-tier hexagonal cell configuration with a wrap-around technique. A UMTS cellular wireless network [17], with a fast power controller running at 1500 updates per second, is simulated. The average cross-correlation between the codes $\left(E \xi^{1}\right)$ is assumed to be 0.5 . Three types of services are used: $12.2 \mathrm{kbps}$ voice (with the required bit energy to the interference spectral density $E_{b} / I_{0}$ of $5 \mathrm{~dB}$ ), $32 \mathrm{kbps}$ data (with $E_{b} / I_{0}$ of $3 \mathrm{~dB}$ ) and $64 \mathrm{kbps}$ data (with $E_{b} / I_{0}$ of $2 \mathrm{~dB}$ ). We assume 5 Erlangs of voice traffic. For data services, we assume a Pareto call duration (see Section II) with $\alpha_{1}=1.5, E \tau_{1}=2 \mathrm{sec}$ and $\alpha_{2}=1.8, E \tau_{2}=1.5 \mathrm{sec}$. The modeling time scale is $T_{w}=10 \mathrm{msec}$. The arrival rates of both data services have a Poisson distribution with an average rate of 10 arrivals per second. Channel fading is based on the Gudmundson model [18] with $\sigma_{c}=8 \mathrm{~dB}$ and $T_{f}=100$ msec. A distance-dependent channel loss with path exponent $\gamma_{c}=-4$ for $c=1, \ldots, C$ is considered. Users of different services are distributed uniformly, and there are no users with soft-hand-off condition. A power-based call admission control mechanism is also applied in the downlink, in which a new arrival is granted if serving that user does not cause the total BS transmitted power to exceed its corresponding maximum value [12]. Simulation parameters are presented in Table I. The heavy-tail call durations of data services satisfy the conditions of Proposition 1, which gives the self-similarity index $H=0.75$.

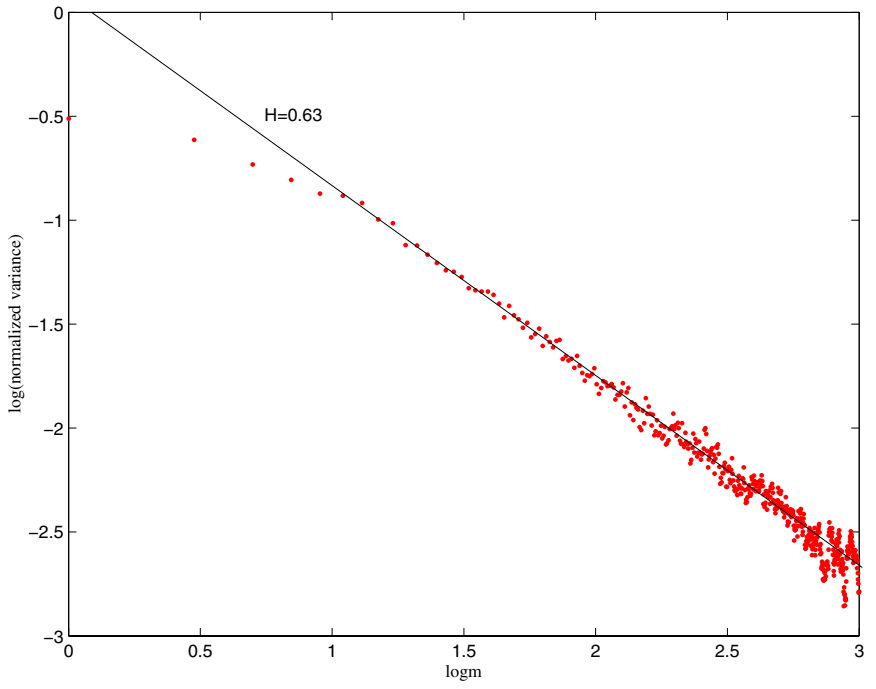

Fig. 1. Normalized variance plot for the total received downlink interference in the simulated network.

In the above configuration, we study the time trace of the received downlink interference measured at different locations. To estimate the self-similarity index $H$, we use the variance plot method in [16], and divide $I$ into non-overlapping blocks each with $m$ samples. For an asymptotic self-similar process with self-similarity index $H$, the variance of the mean processes for $m \rightarrow \infty$ is $L(m) m^{2 H-2}, 0.5<H<1$ [15]. Therefore, in a logarithmic scale, the variance is a straight line. Using the slope of this line, we can estimate $H$. Fig. 1 shows the variance in the logarithmic scale. Using a linear curve fitting, we obtain $H=0.63$, that confirms the self-similarity of the total interference. The discrepancy between the estimated value of $H$ and its value obtained from Proposition 1 is mainly due to the fact that the BS transmitted power exceeds its predetermined value, which results in rejecting some calls.

We also obtain the outage probability for a case with the above setting but instead of the Pareto traffic we have the equivalent exponential call duration distribution with $E \tau_{1}=2$ $\mathrm{s}$ and $E \tau_{2}=1.5 \mathrm{~s}$. The evaluated outage probability for the case of heavy tail traffic is 0.0012 and for the case of heavy tail call duration is 0.0035 that indicates a significant increase in the outage probability.

\section{CONCLUSions}

In this paper, we have studied the effect of users' traffic characteristics on the temporal behavior of total downlink interference in multi-service wireless CDMA networks. We have shown that under certain conditions on traffic characteristics that cover a range of practical situations, the downlink interference follows an asymptotically self-similar process and thus is long-range dependent. The asymptotic outage probability is then obtained and shown that in a network that is designed based on the Poisson assumptions the outage probability is degraded by the interference long range dependent that emanates from its self-similarity. Simulation results for actual cases confirm analytical results. 


\section{ACKNOWLEDGMENT}

The work of K. Navaie and Y. Zhao were supported in part by Nortel Network (Ottawa, Canada), National Capital Institute of Telecommunications (NCIT) (Ottawa, Canada) and Mathematics of Information Technology and Complex Systems (MITACS) (Canada).

\section{REFERENCES}

[1] A. J. Viterbi, CDMA: Principles of Spread Spectrum Communication. Addison-Wesley, 1995

[2] S. Verdu, Multiuser Detection. Cambridge University Press, 1998.

[3] C. C. Chung and S. V. Hanly, "Calculating the outage probability in CDMA network with spatial poisson traffic," IEEE Transactions on Vehicular Technology, vol. 50, pp. 183-204, 2001.

[4] J. Evans and D. Everitt, "On the teletraffic capacity of CDMA cellular networks," IEEE Transactions on Vehicular Technology, vol. 48, pp. 153-165, 1999.

[5] F. P. Kelly, "Loss networks," Annals of Applied Probability, pp. 319378, 1991.

[6] A. Erramilli, M. Roughan, D. Veitch, and W. Willinger, "Self-similar traffic and network dynamics," Proceedings of the IEEE, vol. 90, no. 5, pp. 800-819, 2002.

[7] A. G. Qureshi, "Modelling long-range dependence in multimedia wireless networks," Proceedings of TENCON '97. IEEE Region 10 Annual Conference. Speech and Image Technologies for Computing and Telecommunications, pp. 637-640, 1997.
[8] B. Tsybakov, "Probability of heavy traffic period in third generation CDMA mobile communication," Proceedings of IEEE Workshop on Mobile Multimedia Communications, MoMuC'99, pp. 27-34, 1999.

[9] J. Zhang, M. Hu, and N. B. Shroff, "Bursty data over CDMA: MAI self similarity rate control and admission control," Proceedings of IEEE INFOCOM'02, pp. 391-399, 2002.

[10] J. Zhang and T. Konstantopoulos, "Multi-access interference process is self-similar in multimedia CDMA/ cellular networks," preprint, 2002.

[11] M. S. Taqqu, W. Willinger, and R. Sherman, "Proof of a fundamental result in self-similar traffic modeling," Computer Communication Review, vol. 27, pp. 5-23, 1997.

[12] K. Navaie, S. Valaee, A. R. Sharafat, and E. S. Sousa, "On the downlink interference in heterogeneous wireless DS-CDMA networks," preprint, 2004.

[13] J. Evans and D. Everitt, "Effective bandwidth-based admission control for multiservice CDMA cellular networks," IEEE Transactions on Vehicular Technology, vol. 48, pp. 34-46, 1999.

[14] N. G. Duffield and N. O'Connel, "Large deviations and overflow probabilities for the general single server queue, with applications," Mathematical Proceedings of the Cambridge Philosophical Society, vol. 118, pp. 363-374, 1995.

[15] B. Tsybakov and N. D. Georganas, "Self-similar processes in communications networks," IEEE Transactions on Information Theory, vol. 44, no. 5, pp. 1713-1725, 1999.

[16] J. Beran, Statistics for Long-Memory Processes. New York: Chapman \& Hall, 1994

[17] H. Holma and A. Toskala, WCDMA for UMTS: Radio access for third generation mobile communications. John Willy and Sons, 2000.

[18] G. L. Stuber, Principles of Mobile Communication. Kluwer Academic Publishers, 1996. 